\title{
Analysis of Relationship between Socio-Economic Factors and Subjective Health Condition among the Community Inhabitants of Japan
}

\author{
Keiji Muramatsu'), Shinya Matsuda ${ }^{1,2)}$, Kenshi Hayashida ${ }^{2)}$, \\ Tatsuhiko Kubo ${ }^{1)}$, Yoshihisa Fujino ${ }^{1)}$ \\ ${ }^{1)}$ Department of Preventive Medicine and Community Health, School of Medicine, University of Occupational \\ and Environmental Health \\ ${ }^{2}$ Medical Informatics Division, University Hospital of University of Occupational and Environmental Health, \\ Japan
}

\begin{abstract}
In order to examine the relationship between socio-economic factors and subjective health condition, we have analyzed the data of rural community inhabitants in Japan. We have distributed questionnaires by post for 2,928 households and received answer sheets from 1,580 households (54.0\%). Contents of questionnaire are as follows; demographic data, subjective health status by SF8, lifestyle, social activity, and social capital. The results indicated that persons with better 'economic status' and higher 'frequency of participation to community events' showed statistically significant better subjective health status evaluated by SF8 questionnaires. As previous literatures have indicated, our results also suggested a significant impact of socio-economic factors to health status.
\end{abstract}

Key words: Subjective health status, Socio-economic factors, SF8, Health promotion

\section{* Introduction}

The relationship between socio-economic factors and health status has long been attracting concerns of researchers for the last decades. As the WHO report indicated $^{1)}$, it is a solid fact that persons under the lower socio-economic condition tend to have lower health status. The inequalities in health among groups of socioeconomic status constitute the challenges for public health. Lower mortality and morbidity is associated with almost any positive indicator of socioeconomic status. Accessibility to the health services might be one of the explanations for these correlations. More educated people are better able to understand and use health information might be additional

Received: June 13, 2012

Accepted: August 8, 2012

Correspondence: K. Muramatsu, 1-1 Iseigaoka, Yahatanishiku, Kitakyushu, Fukuoka 807-8555, Japan;

e-mail:km@med.uoeh-u.ac.jp argument. However, it is also possible that people in the lower social class may tend to have unhealthy behavior, such as tobacco smoking, drug abuse, or alcoholism $^{2-4)}$.

Japan has long been thought as a society of less social divide. However, the problem of social divide is becoming a big social problem in Japan because of a long lasting economic slump after the end of bubble economy. In order to overcome the managerial difficulty, most of the Japanese corporations have tried to reduce the personnel cost. They have reduced fulltime employees and replaced them by part-time workers with limited work contract. It is said that this change of working culture has made our society unstable and caused social divide.

In this study, the authors examined the relationship between socio-economic factors and subjective health condition using the data of rural community inhabitants in Japan. 
Table 1 Numner of respondants stratified by sex and age category

\begin{tabular}{|c|c|c|c|c|}
\hline \multirow{2}{*}{\multicolumn{2}{|c|}{ Age category }} & \multicolumn{2}{|c|}{ Sex } & \multirow{3}{*}{$\begin{array}{c}\text { Total } \\
6\end{array}$} \\
\hline & & \multirow{2}{*}{$\begin{array}{c}\text { Male } \\
3\end{array}$} & \multirow{2}{*}{$\begin{array}{c}\text { Female } \\
3\end{array}$} & \\
\hline $20-29$ & $\mathrm{~N}$ & & & \\
\hline & $\%$ & $0.3 \%$ & $0.9 \%$ & $0.4 \%$ \\
\hline $30-39$ & $\mathrm{~N}$ & 63 & 24 & 87 \\
\hline & $\%$ & $5.5 \%$ & $7.0 \%$ & $5.9 \%$ \\
\hline $40-49$ & $\mathrm{~N}$ & 88 & 18 & 106 \\
\hline & $\%$ & $7.7 \%$ & $5.2 \%$ & $7.1 \%$ \\
\hline $50-59$ & $\mathrm{~N}$ & 207 & 41 & 248 \\
\hline & $\%$ & $18.1 \%$ & $11.9 \%$ & $16.7 \%$ \\
\hline $60-69$ & $\mathrm{~N}$ & 361 & 65 & 426 \\
\hline & $\%$ & $31.6 \%$ & $18.8 \%$ & $28.6 \%$ \\
\hline $70-79$ & $\mathrm{~N}$ & 282 & 91 & 373 \\
\hline & $\%$ & $24.7 \%$ & $26.4 \%$ & $25.1 \%$ \\
\hline $80-$ & $\mathrm{N}$ & 138 & 103 & 241 \\
\hline & $\%$ & $12.1 \%$ & $29.9 \%$ & $16.2 \%$ \\
\hline Total & $\mathrm{N}$ & 1142 & 345 & 1487 \\
\hline & $\%$ & $100.0 \%$ & $100.0 \%$ & \\
\hline $100.0 \%$ & & & & \\
\hline
\end{tabular}

\section{Studied Population and Method}

Data were gathered from inhabitants of a rural community of Fukuoka prefecture. We have distributed questionnaires by post for 2928 households and received answer sheets from 1580 households $(54.0 \%)$. Contents of questionnaire are as follows; demographic data, subjective health status by SF8, lifestyle, social activity, and social capital.

Based on the above data, we have analyzed the relationship between the subjective health status and socio-economic factors. All analyses were conducted by SPSS ver. 19 (IBM, Tokyo).

\section{Results}

Table 1 shows the number of respondents according to sex and age category. Male represents $76.8 \%$ $(1,142)$ and female $23.2 \%$ (345). Persons of $60-69$ were the largest proportion (426 persons, $28.6 \%$ ) followed by $70-79$ (373, 25.1\%), 50-59 (248, 16.7\%), and 80 years old and more $(241,16.2 \%)$. More than $70 \%$ of respondents were 60 years old and more.

Table 2 shows the frequency of community events participation stratified by sex and age category.
Usually, female shows higher participation rate, but female inhabitants tend to respond lower participation rate compared with male in this population. As we sent the questionnaire to the head of household, answer might be biased. In the case of household of which female is head, they might be single and possible to work outside household. This situation might decrease the participation rate of community event. Generally speaking, the participation rate increases up to $70-79$ years old and decreases after 80 years old in this population.

Table 3 shows economic status (self evaluation) stratified by sex and age category. Female persons tend to respond "slightly problematic" or "problematic" for all age category compared with male. This might be a result of bias above mentioned. It is interesting that younger generation (under 50 years old) tends to respond "problematic".

Table 4 shows results of subjective health status measured by SF 8 stratified by sex and age category. In all items, male average score tend to be higher than female. Results of ANOVA, we showed statisticallysignificant difference except for Bodily pain (BP), Mental health (MH) and Mental Component Summery (MCS) among female responders. In most items, 
Table 2 Frequency of partcipation at community events

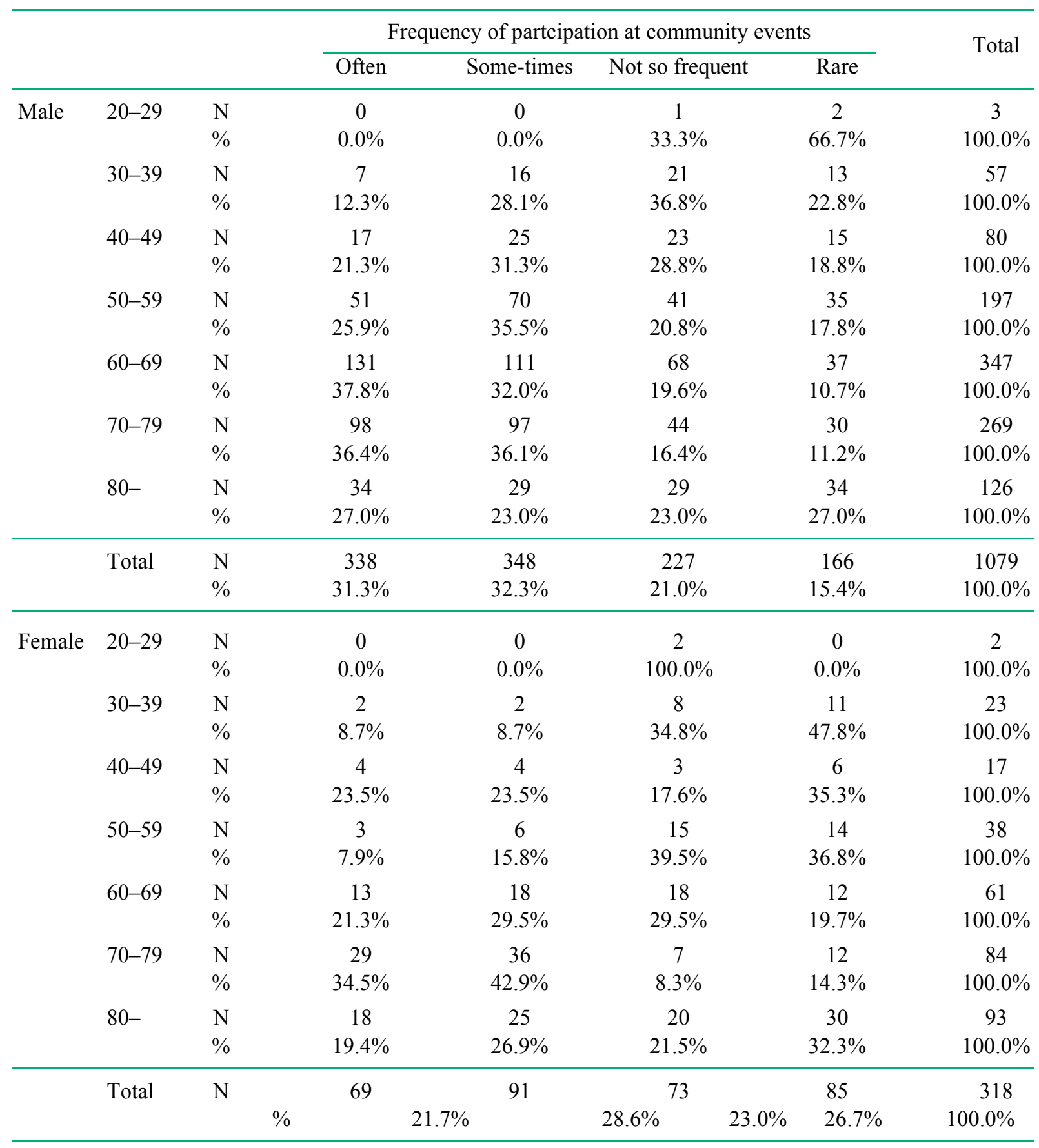

$\mathrm{p}<0.01$; Chi square test

the average score decreases with increasing age however male responders who are at working age were tended to show lower score than over 60 years old people. This result might be due to stress at work as in Survey on State of Employees' Health by MHLW showed: 'The percentage of workers who have anxiety, worry and stress about their work or occupational life had become 58.0\%?.
Table 5 shows the results of correlation analysis. Both 'economic status' and 'frequency of participation to community events' showed statistically significant negative correlations with all of the items of SF 8 among male responders. This result indicated that persons with better 'economic status' and higher 'frequency of participation to community events' showed statistically significant better subjective health status 
Table 3 Economic status stratified by sex and age category (self evaluation)

\begin{tabular}{|c|c|c|c|c|c|c|c|}
\hline \multicolumn{3}{|c|}{ Age category } & \multicolumn{4}{|c|}{ Economic status } & \multirow{3}{*}{$\begin{array}{c}\text { Total } \\
3\end{array}$} \\
\hline & & & \multirow{2}{*}{$\begin{array}{c}\text { No problem } \\
0\end{array}$} & \multirow{2}{*}{$\begin{array}{c}\text { Not so problem } \\
1\end{array}$} & \multirow{2}{*}{$\begin{array}{c}\text { Slightly problematic } \\
2\end{array}$} & \multirow{2}{*}{$\begin{array}{c}\text { Problematic } \\
0\end{array}$} & \\
\hline Male & $20-29$ & $\mathrm{~N}$ & & & & & \\
\hline & & $\%$ & $0.0 \%$ & $33.3 \%$ & $66.7 \%$ & $0.0 \%$ & $100.0 \%$ \\
\hline & $30-39$ & $\mathrm{~N}$ & 8 & 30 & 14 & 10 & 62 \\
\hline & & $\%$ & $12.9 \%$ & $48.4 \%$ & $22.6 \%$ & $16.1 \%$ & $100.0 \%$ \\
\hline & $40-49$ & $\mathrm{~N}$ & 12 & 43 & 21 & 12 & 88 \\
\hline & & $\%$ & $13.6 \%$ & $48.9 \%$ & $23.9 \%$ & $13.6 \%$ & $100.0 \%$ \\
\hline & $50-59$ & $\mathrm{~N}$ & 22 & 104 & 59 & 19 & 204 \\
\hline & & $\%$ & $10.8 \%$ & $51.0 \%$ & $28.9 \%$ & $9.3 \%$ & $100.0 \%$ \\
\hline & $60-69$ & $\mathrm{~N}$ & 47 & 190 & 96 & 24 & 357 \\
\hline & & $\%$ & $13.2 \%$ & $53.2 \%$ & $26.9 \%$ & $6.7 \%$ & $100.0 \%$ \\
\hline & $70-79$ & $\mathrm{~N}$ & 35 & 162 & 61 & 18 & 276 \\
\hline & & $\%$ & $12.7 \%$ & $58.7 \%$ & $22.1 \%$ & $6.5 \%$ & $100.0 \%$ \\
\hline & $80-$ & $\mathrm{N}$ & 24 & 81 & 19 & 9 & 133 \\
\hline & & $\%$ & $18.0 \%$ & $60.9 \%$ & $14.3 \%$ & $6.8 \%$ & $100.0 \%$ \\
\hline & Total & $\mathrm{N}$ & 148 & 611 & 272 & 92 & 1123 \\
\hline & & $\%$ & $13.2 \%$ & $54.4 \%$ & $24.2 \%$ & $8.2 \%$ & $100.0 \%$ \\
\hline \multirow[t]{16}{*}{ Female } & $20-29$ & $\mathrm{~N}$ & 0 & 1 & 2 & 0 & 3 \\
\hline & & $\%$ & $0.0 \%$ & $33.3 \%$ & $66.7 \%$ & $0.0 \%$ & $100.0 \%$ \\
\hline & $30-39$ & $\mathrm{~N}$ & 2 & 9 & 8 & 4 & 23 \\
\hline & & $\%$ & $8.7 \%$ & $39.1 \%$ & $34.8 \%$ & $17.4 \%$ & $100.0 \%$ \\
\hline & $40-49$ & $\mathrm{~N}$ & 2 & 6 & 7 & 3 & 18 \\
\hline & & $\%$ & $11.1 \%$ & $33.3 \%$ & $38.9 \%$ & $16.7 \%$ & $100.0 \%$ \\
\hline & $50-59$ & $\mathrm{~N}$ & 5 & 20 & 11 & 5 & 41 \\
\hline & & $\%$ & $12.2 \%$ & $48.8 \%$ & $26.8 \%$ & $12.2 \%$ & $100.0 \%$ \\
\hline & $60-69$ & $\mathrm{~N}$ & 5 & 25 & 25 & 10 & 65 \\
\hline & & $\%$ & $7.7 \%$ & $38.5 \%$ & $38.5 \%$ & $15.4 \%$ & $100.0 \%$ \\
\hline & $70-79$ & $\mathrm{~N}$ & 13 & 40 & 22 & 12 & 87 \\
\hline & & $\%$ & $14.9 \%$ & $46.0 \%$ & $25.3 \%$ & $13.8 \%$ & $100.0 \%$ \\
\hline & $80-$ & $\mathrm{N}$ & 27 & 47 & 22 & 4 & 100 \\
\hline & & $\%$ & $27.0 \%$ & $47.0 \%$ & $22.0 \%$ & $4.0 \%$ & $100.0 \%$ \\
\hline & Total & $\mathrm{N}$ & 54 & 148 & 97 & 38 & 337 \\
\hline & & $\%$ & $16.0 \%$ & $43.9 \%$ & $28.8 \%$ & $11.3 \%$ & $100.0 \%$ \\
\hline
\end{tabular}

$\mathrm{p}<0.01$; Chi square test

evaluated by SF8 questionnaires Among the female responders, economic status showed statistically negative correlation with subject health status measured by SF8 except for Physical functioning (PF), Role physical (RP) and Physical Component Summary (PCS). On the other hand, frequency of participation to community events showed statistically negative correlation with subject health status measured by SF8 except for Role physical (RP) and Bodily pain (BP).

\section{* Discussion}

As previous literatures have indicated ${ }^{1-4)}$, our results also suggested a significant impact of socioeconomic factors to health status. Several limitations must be considered when interpreting our results. First, this study is a cross-sectional study, so it is difficult to discuss direction of causality of socio-economic factors and subjective health condition. Until now, there are few studies analyzed the relationship between socio-economic status and subjective health 
Table 4 Health status measured by SF8 stratified by sex and age category

\begin{tabular}{|c|c|c|c|c|c|c|c|c|c|c|c|c|}
\hline & $\begin{array}{c}\text { age } \\
\text { category }\end{array}$ & & $\begin{array}{c}\text { General } \\
\text { health } \\
(\mathrm{GH})\end{array}$ & $\begin{array}{l}\text { Physical } \\
\text { function- } \\
\text { ing }(\mathrm{PF})\end{array}$ & $\begin{array}{c}\text { Role } \\
\text { physical } \\
\text { (RP) }\end{array}$ & $\begin{array}{c}\text { Bodily } \\
\text { pain (BP) }\end{array}$ & $\begin{array}{c}\text { Vitality } \\
\text { (VT) }\end{array}$ & $\begin{array}{l}\text { Social } \\
\text { function- } \\
\text { ing }(\mathrm{SF})\end{array}$ & $\begin{array}{c}\text { Mental } \\
\text { health } \\
(\mathrm{MH})\end{array}$ & $\begin{array}{c}\text { Role } \\
\text { emotional } \\
\text { (RE) }\end{array}$ & $\begin{array}{l}\text { Physical } \\
\text { Component } \\
\text { Summary } \\
\text { (PCS) }\end{array}$ & $\begin{array}{c}\text { Mental } \\
\text { Component } \\
\text { Summery } \\
\text { (MCS) }\end{array}$ \\
\hline \multirow[t]{24}{*}{ Male } & \multirow[t]{3}{*}{$20-29$} & $\mathrm{~N}$ & 3 & 3 & 3 & 3 & 3 & 3 & 3 & 3 & 3 & 3 \\
\hline & & Mean & 50.7 & 53.6 & 53.9 & 57.4 & 54.5 & 51.6 & 55.1 & 52.6 & 52.8 & 51.9 \\
\hline & & $\mathrm{SD}$ & 0.0 & 0.0 & 0.0 & 4.9 & 0.0 & 5.5 & 4.1 & 3.0 & 3.0 & 5.6 \\
\hline & \multirow[t]{3}{*}{$30-39$} & $\mathrm{~N}$ & 62 & 62 & 61 & 62 & 61 & 60 & 62 & 62 & 58 & 58 \\
\hline & & Mean & 48.6 & 51.6 & 51.9 & 51.1 & 50.6 & 51.1 & 49.3 & 49.2 & 50.7 & 48.7 \\
\hline & & SD & 7.7 & 6.1 & 4.2 & 9.6 & 6.9 & 7.7 & 7.8 & 6.7 & 6.0 & 7.1 \\
\hline & \multirow[t]{3}{*}{$40-49$} & $\mathrm{~N}$ & 87 & 86 & 85 & 87 & 87 & 83 & 84 & 87 & 77 & 77 \\
\hline & & Mean & 48.6 & 51.8 & 52.3 & 50.4 & 50.0 & 51.5 & 49.5 & 50.1 & 50.6 & 48.8 \\
\hline & & SD & 6.4 & 5.6 & 3.7 & 8.7 & 5.8 & 5.5 & 6.3 & 5.7 & 5.4 & 6.2 \\
\hline & \multirow[t]{3}{*}{$50-59$} & $\mathrm{~N}$ & 204 & 203 & 202 & 205 & 204 & 193 & 204 & 205 & 187 & 187 \\
\hline & & Mean & 49.3 & 50.5 & 50.7 & 50.2 & 50.7 & 50.5 & 50.0 & 49.4 & 49.5 & 49.1 \\
\hline & & SD & 5.9 & 6.8 & 5.6 & 7.6 & 6.0 & 6.9 & 6.3 & 6.3 & 6.1 & 6.3 \\
\hline & \multirow[t]{3}{*}{$60-69$} & $\mathrm{~N}$ & 356 & 356 & 355 & 357 & 354 & 345 & 356 & 358 & 333 & 333 \\
\hline & & Mean & 49.7 & 49.8 & 50.3 & 50.6 & 51.8 & 51.1 & 52.3 & 50.8 & 48.5 & 51.6 \\
\hline & & $\mathrm{SD}$ & 6.4 & 7.0 & 6.9 & 8.0 & 6.1 & 7.3 & 5.8 & 5.7 & 6.5 & 5.3 \\
\hline & \multirow[t]{3}{*}{$70-79$} & $\mathrm{~N}$ & 265 & 264 & 261 & 266 & 260 & 247 & 265 & 265 & 236 & 236 \\
\hline & & Mean & 48.1 & 47.7 & 47.3 & 48.8 & 49.8 & 49.8 & 51.1 & 49.2 & 46.1 & 50.9 \\
\hline & & SD & 7.4 & 8.6 & 9.2 & 8.9 & 7.8 & 7.7 & 6.4 & 8.6 & 7.9 & 6.2 \\
\hline & \multirow[t]{3}{*}{$80-$} & $\mathrm{N}$ & 130 & 126 & 124 & 127 & 130 & 122 & 128 & 127 & 114 & 114 \\
\hline & & Mean & 46.0 & 43.2 & 42.8 & 47.9 & 47.8 & 45.8 & 50.5 & 46.6 & 42.1 & 49.6 \\
\hline & & SD & 7.5 & 11.6 & 11.5 & 8.6 & 7.6 & 10.9 & 7.2 & 11.2 & 9.5 & 8.1 \\
\hline & \multirow[t]{3}{*}{ Total } & $\mathrm{N}$ & 1107 & 1100 & 1091 & 1107 & 1099 & 1053 & 1102 & 1107 & 1008 & 1008 \\
\hline & & Mean & 48.7 & 48.9 & 49.1 & 49.8 & 50.5 & 50.1 & 51.0 & 49.5 & 47.7 & 50.4 \\
\hline & & SD & 6.9 & 8.2 & 8.1 & 8.4 & 6.8 & 7.9 & 6.5 & 7.5 & 7.5 & 6.3 \\
\hline \multicolumn{3}{|c|}{ p-value (ANOVA) } & $<0.001$ & $<0.001$ & $<0.001$ & $<0.001$ & $<0.001$ & $<0.001$ & $<0.001$ & $<0.001$ & $<0.001$ & $<0.001$ \\
\hline \multirow[t]{24}{*}{ Female } & \multirow[t]{3}{*}{$20-29$} & $\mathrm{~N}$ & 3 & 3 & 3 & 3 & 3 & 3 & 3 & 3 & 3 & 3 \\
\hline & & Mean & 44.3 & 48.0 & 46.5 & 43.4 & 46.5 & 46.1 & 50.9 & 49.3 & 42.8 & 50.0 \\
\hline & & SD & 5.5 & 5.9 & 3.4 & 4.8 & 4.3 & 8.2 & 6.3 & 4.9 & 5.0 & 6.0 \\
\hline & \multirow[t]{3}{*}{$30-39$} & $\mathrm{~N}$ & 49 & 23 & 23 & 24 & 24 & 23 & 24 & 23 & 22 & 22 \\
\hline & & Mean & 48.6 & 51.7 & 50.8 & 48.3 & 50.5 & 44.7 & 47.6 & 48.9 & 50.2 & 46.4 \\
\hline & & SD & 7.4 & 3.7 & 5.4 & 9.6 & 5.0 & 11.8 & 9.1 & 6.6 & 5.2 & 8.9 \\
\hline & \multirow[t]{3}{*}{$40-49$} & $\mathrm{~N}$ & 18 & 18 & 18 & 18 & 18 & 16 & 18 & 18 & 16 & 16 \\
\hline & & Mean & 48.4 & 51.8 & 50.2 & 50.9 & 48.5 & 49.3 & 50.1 & 49.6 & 49.2 & 47.7 \\
\hline & & SD & 7.1 & 4.8 & 9.2 & 8.1 & 8.5 & 8.6 & 8.9 & 7.2 & 7.0 & 9.3 \\
\hline & \multirow[t]{3}{*}{$50-59$} & $\mathrm{~N}$ & 41 & 41 & 41 & 41 & 41 & 39 & 41 & 41 & 39 & 39 \\
\hline & & Mean & 49.3 & 50.6 & 51.1 & 47.4 & 49.3 & 51.0 & 49.3 & 49.8 & 48.5 & 49.4 \\
\hline & & SD & 6.3 & 4.0 & 4.4 & 8.1 & 5.8 & 5.5 & 8.2 & 5.6 & 5.3 & 7.0 \\
\hline & \multirow[t]{3}{*}{$60-69$} & $\mathrm{~N}$ & 64 & 64 & 62 & 64 & 63 & 58 & 64 & 63 & 57 & 57 \\
\hline & & Mean & 48.2 & 48.7 & 49.3 & 48.2 & 51.0 & 48.9 & 49.6 & 49.1 & 47.2 & 49.2 \\
\hline & & SD & 7.6 & 7.2 & 7.0 & 10.0 & 7.1 & 8.4 & 7.7 & 7.8 & 7.3 & 7.4 \\
\hline & \multirow[t]{3}{*}{$70-79$} & $\mathrm{~N}$ & 88 & 85 & 87 & 88 & 86 & 82 & 88 & 87 & 75 & 75 \\
\hline & & Mean & 47.9 & 46.3 & 47.6 & 47.8 & 50.4 & 47.6 & 50.0 & 48.6 & 45.3 & 49.9 \\
\hline & & SD & 6.5 & 7.4 & 9.1 & 8.4 & 7.2 & 9.0 & 6.9 & 8.1 & 8.0 & 7.2 \\
\hline & $80-$ & $\mathrm{N}$ & 95 & 95 & 81 & 94 & 95 & 90 & 94 & 93 & 84 & 84 \\
\hline & & Mean & 45.4 & 39.8 & 39.9 & 46.8 & 46.1 & 44.0 & 47.7 & 43.1 & 40.3 & 47.2 \\
\hline & & SD & 7.7 & 13.2 & 13.1 & 8.7 & 8.2 & 11.8 & 8.4 & 13.0 & 10.3 & 8.8 \\
\hline & Total & $\mathrm{N}$ & 333 & 329 & 329 & 332 & 330 & 311 & 332 & 328 & 296 & 296 \\
\hline & & Mean & 47.5 & 46.1 & 46.5 & 47.7 & 49.0 & 47.1 & 49.0 & 47.4 & 45.2 & 48.5 \\
\hline & & $\mathrm{SD}$ & 7.2 & 9.9 & 10.3 & 8.8 & 7.5 & 9.9 & 7.9 & 9.7 & 8.7 & 8.0 \\
\hline p-value & ANOVA) & & 0.040 & $<0.001$ & $<0.001$ & 0.633 & 0.001 & 0.003 & 0.480 & $<0.001$ & $<0.001$ & 0.294 \\
\hline & & & 1.207 & 2.828 & 2.520 & 2.072 & 1.479 & 2.980 & 1.999 & 2.176 & 2.523 & 1.856 \\
\hline
\end{tabular}


Table 5 Correlation coeficients (CC) among the socio-economic factors and items of SF8 questionnaire

\begin{tabular}{|c|c|c|c|c|c|c|c|c|c|c|c|c|c|c|}
\hline & & & $\begin{array}{l}\text { Eco- } \\
\text { nomic } \\
\text { status }\end{array}$ & $\begin{array}{c}\text { Commu- } \\
\text { nity event } \\
\text { partipation }\end{array}$ & $\begin{array}{l}\text { General } \\
\text { health } \\
(\mathrm{GH})\end{array}$ & $\begin{array}{l}\text { Physical } \\
\text { function- } \\
\text { ing (PF) }\end{array}$ & $\begin{array}{c}\text { Role } \\
\text { physical } \\
\text { (RP) }\end{array}$ & $\begin{array}{c}\text { Bodily } \\
\text { pain (BP) }\end{array}$ & $\begin{array}{l}\text { Vitality } \\
\text { (VT) }\end{array}$ & $\begin{array}{l}\text { Social } \\
\text { function- } \\
\text { ing SF) }\end{array}$ & $\begin{array}{l}\text { Mental } \\
\text { health } \\
(\mathrm{MH})\end{array}$ & $\begin{array}{c}\text { Role } \\
\text { emotional } \\
\text { (RE) }\end{array}$ & $\begin{array}{l}\text { Physical } \\
\text { Component } \\
\text { Summary } \\
\text { (PCS) }\end{array}$ & $\begin{array}{c}\text { Mental } \\
\text { Component } \\
\text { Summery } \\
\text { (MCS) }\end{array}$ \\
\hline \multirow[t]{35}{*}{ Male } & \multirow{3}{*}{ Economic status } & $\mathrm{CC}$ & 1 & 0.091 & -0.207 & -0.161 & -0.200 & -0.176 & -0.243 & $3-0.151$ & -0.227 & -0.223 & -0.171 & -0.210 \\
\hline & & p-value & & 0.003 & 0.000 & 0.000 & 0.000 & 0.000 & 0.000 & 0.000 & 0.000 & 0.000 & 0.000 & 0.000 \\
\hline & & $\mathrm{n}$ & 1133 & 1073 & 1100 & 1094 & 1089 & 1102 & 1093 & 1048 & 1097 & 1102 & 1006 & 100 \\
\hline & \multirow{3}{*}{$\begin{array}{l}\text { Community } \\
\text { event partipation }\end{array}$} & $\mathrm{CC}$ & & 1 & -0.184 & -0.172 & -0.141 & -0.121 & -0.223 & $3-0.196$ & -0.164 & -0.193 & -0.121 & -0.20 \\
\hline & & p-value & & & 0.000 & 0.000 & 0.000 & 0.000 & 0.000 & 0.000 & 0.000 & 0.000 & 0.000 & 0.00 \\
\hline & & $\mathrm{n}$ & & 1088 & 1057 & 1054 & 1045 & 1058 & 1049 & 1007 & 1055 & 1058 & 967 & 96 \\
\hline & \multirow{3}{*}{$\begin{array}{l}\text { General health } \\
(\mathrm{GH})\end{array}$} & $\mathrm{CC}$ & & & 1 & 0.464 & 0.496 & 0.457 & 0.669 & 0.428 & 0.420 & 0.464 & 0.612 & 0.38 \\
\hline & & p-value & & & & 0.000 & 0.000 & 0.000 & 0.000 & 0.000 & 0.000 & 0.000 & 0.000 & 0.000 \\
\hline & & $\mathrm{n}$ & & & 1117 & 1103 & 1095 & 1110 & 1101 & 1057 & 1106 & 1110 & 1018 & 1018 \\
\hline & \multirow{3}{*}{$\begin{array}{l}\text { Physical func- } \\
\text { tioning (PF) }\end{array}$} & $\mathrm{CC}$ & & & & 1 & 0.793 & 0.526 & 0.477 & 0.530 & 0.312 & 0.469 & 0.791 & 0.17 \\
\hline & & $\mathrm{p}$-value & & & & & 0.000 & 0.000 & 0.000 & 0.000 & 0.000 & 0.000 & 0.000 & 0.00 \\
\hline & & $\mathrm{n}$ & & & & 1110 & 1092 & 1105 & 1096 & 1054 & 1102 & 1107 & 1018 & 1018 \\
\hline & \multirow{3}{*}{$\begin{array}{l}\text { Role physical } \\
\text { (RP) }\end{array}$} & $\mathrm{CC}$ & & & & & 1 & 0.527 & 0.531 & 0.567 & 0.368 & 0.532 & 0.775 & 0.273 \\
\hline & & p-value & & & & & & 0.000 & 0.000 & 0.000 & 0.000 & 0.000 & 0.000 & 0.000 \\
\hline & & $\mathrm{n}$ & & & & & 1101 & 1098 & 1086 & 1047 & 1095 & 1100 & 1018 & 1018 \\
\hline & \multirow[t]{3}{*}{ Bodily pain (BP) } & $\mathrm{CC}$ & & & & & & 1 & 0.453 & 0.388 & 0.384 & 0.428 & 0.754 & 0.173 \\
\hline & & $\mathrm{p}$-value & & & & & & & 0.000 & 0.000 & 0.000 & 0.000 & 0.000 & 0.000 \\
\hline & & $\mathrm{n}$ & & & & & & 1117 & 1104 & 1059 & 1109 & 1115 & 1018 & 1018 \\
\hline & Vitality (VT) & $\mathrm{CC}$ & & & & & & & 1 & 0.442 & 0.400 & 0.463 & 0.598 & 0.443 \\
\hline & & p-value & & & & & & & & 0.000 & 0.000 & 0.000 & 0.000 & 0.000 \\
\hline & & $\mathrm{n}$ & & & & & & & 1109 & 1049 & 1098 & 1103 & 1018 & 1018 \\
\hline & Social function- & $\mathrm{CC}$ & & & & & & & & 1 & 0.495 & 0.613 & 0.420 & 0.624 \\
\hline & ing SF) & $\mathrm{p}$-value & & & & & & & & & 0.000 & 0.000 & 0.000 & 0.000 \\
\hline & & $\mathrm{n}$ & & & & & & & & 1063 & 1059 & 1061 & 1018 & 1018 \\
\hline & Mental health & $\mathrm{CC}$ & & & & & & & & & 1 & 0.649 & 0.146 & 0.865 \\
\hline & $(\mathrm{MH})$ & $\mathrm{p}$-value & & & & & & & & & & 0.000 & 0.000 & 0.000 \\
\hline & & $\mathrm{n}$ & & & & & & & & & 1112 & 1110 & 1018 & 1018 \\
\hline & Role emotional & $\mathrm{CC}$ & & & & & & & & & & 1 & 0.319 & 0.76 \\
\hline & (RE) & $\mathrm{p}$-value & & & & & & & & & & & 0.000 & 0.0 \\
\hline & & $\mathrm{n}$ & & & & & & & & & & 1117 & 1018 & 1018 \\
\hline & Physical Compo- & $\mathrm{CC}$ & & & & & & & & & & & 1 & -0.010 \\
\hline & nent Summary & $\mathrm{p}$-value & & & & & & & & & & & & 0.74 \\
\hline & (PCS) & $\mathrm{n}$ & & & & & & & & & & & 1018 & 1018 \\
\hline & Mental Compo- & $\mathrm{CC}$ & & & & & & & & & & & & \\
\hline & $\begin{array}{l}\text { nent Summery } \\
\text { (MCS) }\end{array}$ & $\underset{n}{p \text {-value }}$ & & & & & & & & & & & & 1018 \\
\hline Female & Economic status & $\mathrm{CC}$ & 1 & 0.148 & -0.205 & -0.009 & -0.023 & -0.179 & -0.204 & -0.148 & -0.278 & -0.208 & -0.018 & -0.276 \\
\hline & & $\mathrm{p}$-value & & 0.008 & 0.000 & 0.871 & 0.679 & 0.001 & 0.000 & 0.009 & 0.000 & 0.000 & 0.759 & 0.000 \\
\hline & & $\mathrm{n}$ & 345 & 323 & 334 & 330 & 328 & 333 & 331 & 312 & 333 & 329 & 298 & 298 \\
\hline & Community & $\mathrm{CC}$ & & 1 & -0.276 & -0.122 & -0.111 & -0.091 & -0.319 & -0.223 & -0.234 & -0.204 & -0.139 & -0.24 \\
\hline & event partipation & $\mathrm{p}$-value & & & 0.000 & 0.031 & 0.051 & 0.107 & 0.000 & 0.000 & 0.000 & 0.000 & 0.019 & 0.000 \\
\hline & & $\mathrm{n}$ & & 327 & 316 & 313 & 311 & 316 & 314 & 297 & 316 & 312 & 284 & 284 \\
\hline & General health & $\mathrm{CC}$ & & & 1 & 0.566 & 0.583 & 0.464 & 0.704 & 0.500 & 0.468 & 0.538 & 0.642 & 0.456 \\
\hline & $(\mathrm{GH})$ & p-value & & & & 0.000 & 0.000 & 0.000 & 0.000 & 0.000 & 0.000 & 0.000 & 0.000 & 0.000 \\
\hline & & $\mathrm{n}$ & & & 342 & 337 & 334 & 339 & 337 & 319 & 340 & 336 & 305 & 305 \\
\hline & Physical func- & $\mathrm{CC}$ & & & & 1 & 0.809 & 0.502 & 0.547 & 0.581 & 0.401 & 0.628 & 0.866 & 0.317 \\
\hline & tioning $(\mathrm{PF})$ & $\mathrm{p}$-value & & & & & 0.000 & 0.000 & 0.000 & 0.000 & 0.000 & 0.000 & 0.000 & 0.000 \\
\hline & & $\mathrm{n}$ & & & & 338 & 332 & 336 & 334 & 316 & 335 & 332 & 305 & 305 \\
\hline & Role physical & $\mathrm{CC}$ & & & & & 1 & 0.492 & 0.569 & 0.651 & 0.484 & 0.698 & 0.816 & 0.445 \\
\hline & (RP) & p-value & & & & & & 0.000 & 0.000 & 0.000 & 0.000 & 0.000 & 0.000 & 0.000 \\
\hline & & $\mathrm{n}$ & & & & & 335 & 334 & 331 & 315 & 333 & 332 & 305 & 305 \\
\hline & Bodily pain (BP) & $\mathrm{CC}$ & & & & & & 1 & 0.491 & 0.413 & 0.428 & 0.505 & 0.708 & 0.269 \\
\hline & & $\mathrm{p}$-value & & & & & & & 0.000 & 0.000 & 0.000 & 0.000 & 0.000 & 0.000 \\
\hline & & $\mathrm{n}$ & & & & & & 341 & 337 & 319 & 339 & 335 & 305 & 305 \\
\hline & Vitality (VT) & $\mathrm{CC}$ & & & & & & & 1 & 0.517 & 0.477 & 0.592 & 0.613 & 0.539 \\
\hline & & p-value & & & & & & & & 0.000 & 0.000 & 0.000 & 0.000 & 0.000 \\
\hline & & $\mathrm{n}$ & & & & & & & 339 & 317 & 336 & 332 & 305 & 30 \\
\hline & Social function- & $\mathrm{CC}$ & & & & & & & & 1 & 0.595 & 0.694 & 0.496 & 0.724 \\
\hline & ing SF) & $\mathrm{p}$-value & & & & & & & & & 0.000 & 0.000 & 0.000 & 0.000 \\
\hline & & $\mathrm{n}$ & & & & & & & & 320 & 319 & 315 & 305 & 305 \\
\hline & Mental health & $\mathrm{CC}$ & & & & & & & & & 1 & 0.684 & 0.251 & 0.8 \\
\hline & $(\mathrm{MH})$ & $\mathrm{p}$-value & & & & & & & & & & 0.000 & 0.000 & 0.00 \\
\hline & & $\mathrm{n}$ & & & & & & & & & 341 & 336 & 305 & 3 \\
\hline & Role emotional & $\mathrm{CC}$ & & & & & & & & & & 1 & 0.507 & 0.7 \\
\hline & (RE) & $\mathrm{p}$-value & & & & & & & & & & & 0.000 & 0.000 \\
\hline & & $\mathrm{n}$ & & & & & & & & & & 337 & 305 & 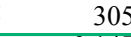 \\
\hline & Physical Compo- & $\mathrm{CC}$ & & & & & & & & & & & 1 & 0.147 \\
\hline & nent Summary & $\mathrm{p}$-value & & & & & & & & & & & & 0.010 \\
\hline & (PCS) & $\mathrm{n}$ & & & & & & & & & & & 305 & 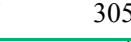 \\
\hline & $\begin{array}{l}\text { Mental Compo- } \\
\text { nent Summery } \\
\text { (MCS) }\end{array}$ & $\begin{array}{c}\text { CC } \\
\text { p-value } \\
n\end{array}$ & & & & & & & & & & & & 3 \\
\hline
\end{tabular}


condition in a longitudinal design ${ }^{5}$. In a recent study, Klein et al. showed that social relations are an important explanatory factor for health inequalities in a longitudinal design ${ }^{5}$. However, the effectiveness of intervention is unclear, therefore we have to organize social programs targeting not only to health promotion but also to community development.

So far as the causal pathway, there have been several theories. Fuchs indicated the difference of time preference would cause different attitude for healthy life style ${ }^{6}$. He has suggested that a person with higher time preference tends to invest for future and as a result not to take unhealthy life style, such as smoking and heavy drinking. A better time preference is associated with more invest in education for oneself and one's children which might explain a positive relationship among educational, economic and health status.

According to our previous study using the Family Income and Expenditure Survey in Japan ${ }^{7)}$, the elasticity of education has been consistently greater than unity. This result suggested that education is very important demand for Japanese household. By contrast, an increase in income leads to a fall in the tobacco smoking suggesting that people living in the household of lower socioeconomic status were likely to consume more tobacco. This result might support the Fuchs' theory on the relationship between education and health status.

Considering the economic difficulty to which most of the local municipalities face, it is rather difficult to largely expand social budget for supporting persons with socio-economic difficulty. One of the possible solutions is to develop a workfare type of social program. The low economic situation during 20 to 60 years old has a severe effect on the socio-economic status of old age period because of lower pen- sion (no pension is also possible) and possibly worse health status. As the experiences of other countries such as Nordic countries, the workfare type of social programs show a promised effect on workability. It is strongly recommended for the Japanese government to promote this type of program in order to decrease the social divide.

\section{References}

1) Wilkinson RG and Marmot M. (eds). Social Determinants of Health; The Solid Facts, 2nd editions, Geneva, WHO, 2003.

2) Mackenbach JP, Stirbu I, Roskam AJ, et al.: Socioeconomic inequalities in health in 22 European countries. N Engl J Med 358, 2468-2481 (2008).

3) Marmot M.: Social determinants of health inequalities. Lancet 365, 1099-1104 (2005).

4) Fukuda $Y$, Nakamura K, Takano T.: Accumulation of health risk behaviours is associated with lower socioeconomic status and women's urban residence: a multilevel analysis in Japan. BMC Public Health 5,53 (2005).

5) Klein J , Vonneilich N , Baumeister SE et al.: Do social relations explain health inequalities? Evidence from a longitudinal survey in a changing eastern German region.: Int J Public Health 57, 619-627 (2012).

6) Fuchs, Victor R. "Time Preference and Health: An Exploratory Study." Economic Aspects of Health, edited by Victor R. Fuchs, pp. 93-120. Chicago: University of Chicago Press, 1982.

7) Yano J, Pham TM, Matsuda S.: The income elasticity of education and tobacco smoking in Japan: The Family Income and Expenditure Survey during the 2000-05 year period, APJDM 4, 83-87 (2010). 